\title{
Cultura en la formación de profesores de lenguas de contexto iberoamericano
}

\author{
Fernanda Tonelli (D) @ \\ Universidade Estadual Paulista (Unesp), Brasil
}

Resumen. El texto tiene como objetivo discutir el componente cultural en la formación de profesores de español y portugués de lenguas extranjeras. A través de una investigación cualitativa, se propone analizar cómo dos carreras de grado de formación de profesores de lenguas -la Licenciatura em Letras - Espanhol, ubicada en Brasil, y el Profesorado de Portugués, en Argentina-, representan la cultura en la formación docente. El estudio se realizará a través del análisis de documentos oficiales que orientan las carreras, es decir, el Plan de Estudios del Profesorado de Portugués del grado argentino; y, el Projeto Pedagógico y los Programas de Estudio de las asignaturas del grado brasileño. Los datos analizados indican que las carreras seleccionadas se apoyan en una concepción tradicional de cultura, marcada por el esencialismo y expresada por el trabajo con la historia de las naciones y la descripción de sus sociedades. Los dos contextos se diferencian por el mayor enfoque dado a la cultura y América Latina (representada por Brasil) por parte de la carrera argentina. Por otro lado, el contexto brasileño está marcado por el silenciamiento de la cultura de la América Latina, lo que indica la herencia colonialista en la formación académica y profesional del profesor de lenguas.

Palabras clave: cultura; formación de profesores; portugués lengua extranjera; español lengua extranjera.

\section{A cultura na formação de professores de línguas no contexto ibero-americano}

Resumo. Este texto tem como objetivo discutir o componente cultural na formação de professores de espanhol e de português línguas estrangeiras. Por meio de uma pesquisa qualitativa, propõe-se analisar de que maneira dois cursos de graduação para a formação de professores de línguas - a Licenciatura em Letras - Espanhol, localizado no Brasil, e a de Português, na Argentina - representam a cultura na formação de professores. Isso será feito mediante a análise de documentos oficiais que orientam os cursos de graduação, ou seja, o Projeto Político Pedagógico e os Programas de Estudo das disciplinas do currículo brasileiro e do argentino. Os dados analisados indicam que os cursos de graduação selecionadas se baseiam em uma concepção tradicional de cultura, marcada pelo essencialismo e manifestada pelo trabalho com a história das nações e a descrição de suas sociedades. Os dois contextos são diferenciados pelo maior enfoque dado à cultura e à América Latina (representada pelo Brasil) pela graduação argentina. Por outro lado, o contexto brasileiro é marcado pelo silenciamento da cultura da América Latina, o que indica a herança colonialista na formação acadêmica e profissional do professor de línguas:

Palavras-chave: cultura; formação de professores; português língua estrangeira; espanhol língua estrangeira

\section{Culture in the training for language teachers in Ibero-American context}

Abstract. This text aims to discuss the cultural component in teacher training courses of Spanish and Portuguese foreign languages. Through qualitative research, it is proposed to analyze how two training courses for language teachers - the course of Licenciatura em Letras - Espanhol, located in Brazil, and Profesorado de Portugués, in Argentina - represent the culture. This is done through the analysis of official documents that guide the courses, that is, the Pedagogical Political Project and the syllabus of the Brazilian course and the Course Guide of the Argentine course. The data analyzed indicate that the courses are based on a traditional conception of culture, marked by essentialism and Eurocentrism and expressed by working with the history of nations and the description of their societies. The two contexts are differentiated by the greater focus given to culture and Latin America (represented by Brazil) by the Argentine course. The Brazilian context is marked by the silencing of Latin American culture, which indicates the colonialist heritage in the academic and professional formation of the language teacher.

Keywords: culture; teacher training; Portuguese Foreign Language; Spanish Foreign Language 


\section{Introducción}

Entre las diversas iniciativas emprendidas para la promoción del portugués y del español como lenguas extranjeras en el contexto iberoamericano de las últimas décadas, se destacan las leyes de Brasil y Argentina que hacen que sea obligatoria la oferta, respectivamente, del español y portugués en las redes básicas de enseñanza de estos países ${ }^{1}$. La proposición de esas lenguas en los currículos de las redes básicas de enseñanza de los dos países en cuestión, trae consigo el aumento de la demanda de profesores para esas asignaturas. Como resultado, en dichos países, hubo una ampliación de la oferta en las carreras de formación de profesores de lenguas.

En lo referente a esta cuestión, se puede observar que tanto en Brasil como en Argentina las directrices educativas para la formación de profesores, orientan la preparación de profesionales para que esté comprometida con una educación de calidad, constituida, entre otros factores, por la valorización cultural. Como ejemplo, podemos citar los Lineamientos Curriculares Nacionales para la Formación Docente Inicial'2, los cuales contienen indicaciones para la organización de carreras de formación de profesores en Argentina. Según este documento, la docencia debe ser considerada como práctica de mediación cultural reflexiva y crítica (Argentina, 2007). Por su parte, en

212 Brasil, las Diretrizes Curriculares Nacionais para a Formação de Professores da Educação Básica, em Cursos de Nível Superior ${ }^{3}$, considera que uno de los papeles que el docente debe atribuirse, es la de incentivar entre los alumnos actividades de enriquecimiento cultural (Brasil, 2002, p. 4). Es importante tener en cuenta que, aunque en ambas directrices el término "cultura" no está claramente definido, el hecho de que esté presente a lo largo de dichos documentos, demuestra el reconocimiento y el entendimiento que la formación de profesores en los contextos indicados, se dará mediante la inserción de la dimensión cultural en las prácticas educativas de estos dos países.

Sin embargo, a pesar de haber consenso entre esos órganos oficiales sobre la importancia del componente cultural en la educación, todavía son escasos los trabajos académicos dedicados a investigar cuáles son las

${ }^{1}$ En Brasil, entró en vigor la Ley 11.161 / 05. En el año 2010, se conoció como "Ley del Español", a partir de 2010, la oferta de lengua española para las escuelas de Enseñanza Media, siendo de carácter optativo la matrícula del alumno. En 2009, en los mismos moldes de oferta obligatoria y matriculación optativa, fue aprobada en Argentina la Ley 26468/2009. La adecuación de las escuelas a esa Ley debería ser hecha hasta 2016. Desde el 16 de febrero de 2017, la Ley brasileña 11.161 / 05 fue revocada por la Ley 13.415 / 2017.

${ }^{2}$ Recuperado de https://bit.ly/2zsA3Bz

${ }^{3}$ Recuperado de https://bit.ly/1DB74aZ 
comprensiones que las carreras de formación de profesores tienen en el contexto de MERCOSUR y sus estudiantes sobre el hacer docente. Al respecto, Dumas (2010) afirma:

Podemos defender que o professor das línguas oficiais do MERCOSUL tem um papel preponderante, já que a língua passa a ser uma das principais chaves de leitura da realidade do Cone Sul e da América Latina, ocupando ali não apenas um papel de transmissores da língua mas, sobretudo, de atores que possam desencadear um processo de conscientização e transformação social. Mas como essa consciência se constrói ou se está construindo? O que de concreto se está realizando para uma mudança efetiva na concepção de integração e do papel dos atores envolvidos? É possível perceber/esboçar a passagem de declarações e propostas grandiosas de intenções a um momento de um fazer reflexivo? (Dumas, 2010, p. 773)

Ante esta situación, nos interesa comprender cómo una carrera de formación de profesores de lengua extranjera de portugués y otra de español en los dos contextos -argentino y brasileño- entienden la cultura como contenido de formación docente. Para ello, seleccionamos dos instituciones habilitadas que forman profesores de lenguas. La Universidad Estadual Paulista (UNESP) de Brasil, que imparte la carrera de Licenciatura em Letras com habilitação em Espanhol Língua Estrangeira; y, la Universidad Nacional de Córdoba (UNC) de Argentina en la que se imparte la carrera del Profesorado de Portugués. Para ello analizaremos algunos documentos oficiales que orientan estas carreras, teniendo como foco el lugar dado a la cultura en esos dispositivos legales.

\section{Sobre la cultura en la formación del profesor de lenguas}

El tratamiento de las culturas en el proceso de enseñanza/aprendizaje es un tema recurrente en la educación. Por mucho tiempo (y aún hoy) se busca una forma -sea un método, un enfoque, principios- que orienten a los profesores a "enseñar cultura". Más recientemente, sin embargo, con la comprensión sobre las dinámicas interpersonales en el actual contexto posmoderno, la discusión sobre cómo enseñar cultura viene siendo desplazada desde una perspectiva homogénea, totalizadora y singular (la cultura), hacia una visión plural, que considera cuestiones como quéculturas, de quién, para quién, además del propio cuestionamiento sobre por qué de considerar la dimensión cultural en el aula y su didáctica (¿cultura es algo que se enseña?). De la misma manera, al pensar críticamente las elecciones que subyacen al campo de los estudios de/sobre/con culturas, es necesario estar consciente 
sobre las no-elecciones, es decir, sobre las identidades culturales que son silenciadas por medio del proceso de selección de determinadas muestras de culturas hegemónicas.

La formación histórica de América Latina está marcada por un silencio físico y simbólico del otro. La esclavitud, el genocidio de los indígenas y la explotación de los más vulnerables, como los pobres y los inmigrantes, y la invisibilidad de saberes llegados de esos grupos, con la consecuente valorización de un tipo de ser y de saber, dio los contornos de una sociedad que se construye a partir de la negación de la alteridad en nombre de una cultura dominante. De acuerdo con Candau (2008b), "o debate multicultural na América Latina nos coloca diante da nossa própria formação histórica, da pergunta sobre como nos construímos socioculturalmente, o que negamos e silenciamos, o que afirmamos, valorizamos e integramos na cultura hegemônica" (p. 17).

Al reflexionar también sobre multiculturalismo y alteridad, Moreira (2001) se pregunta: ¿hemos tenido éxito en el esfuerzo por comprender el mundo del otro? (p. 76). Su inquietud se basa en la idea de que el diálogo entre identidades culturales diferentes, presupone diferentes niveles de poder que sus participantes ocupan. De ese modo, el diálogo, por sí solo, no es capaz de transformar la sociedad si no viene acompañado del cuestionamiento sobre los parámetros que sostienen las desigualdades. En las palabras de Moreira (2001):

É no encontro, no atrito, entre as vozes hegemônicas e as vozes dos sujeitos oprimidos que reside a possibilidade de crítica e desconstrução das representações vigentes das relações sociais e dos grupos culturais, bem como de sua substituição por imagens e representações alternativas que expressem novas representações do mundo em que vivemos, dos grupos com que convivemos e das relações que estabelecemos. (Moreira, 2001, p. 18)

Para Candau (2008a), las diferencias culturales no son algo que debe ser superado, sino las desigualdades. La "superación" de las diferencias culturales implica la homogeneización de la sociedad, con supresión de identidades culturales subalternas en nombre de las que rigen el sistema colonialista. Por su parte, la superación de las desigualdades, emprendida por medio del diálogo entre diferentes grupos socioculturales con el objetivo de reconocer las diferencias, puede ser capaz de llevar a la promoción de la justicia social, cognitiva, económica y cultural.

La escuela, como espacio social, concentra esa diversidad de culturas, que se chocan y se superponen continuamente. En ese sentido, es fundamental que la comunidad escolar esté formada para una postura 
crítica, que rompa con la tradicional homogeneización cultural. Según Candau (2008b), "a escola como instituição está construída tendo por base a afirmação de conhecimentos considerados universais, uma universalidade muitas vezes formal que, se aprofundarmos um pouco, termina por estar assentada na cultura ocidental e européia, considerada como portadora da universalidade" (Candau, 2008b, p. 33).

En relación a la formación de profesores de idiomas, nuestro foco de trabajo, la universalidad de ideas y prácticas tradicionalmente se apoya en la perspectiva epistemológica colonialista, en la que "mantém-se a ideia de uma formação de alta cultura, de estudos superiores, que está na sua origem: os estudos literários aproximados à própria arte da escrita literária e os estudos filológicos relacionados à erudição" (Daher y Sant'Anna, 2010, p. 56). Como resultado, tenemos una formación densa en contenidos académicos relacionados al área de Letras provenientes del Norte global, pero que poco dialoga con la reflexión y la práctica pedagógica pensada desde el Sur global.

En Brasil, las carreras de formación profesional, en general son planeadas y ejecutadas por el área de Educación, por medio de asignaturas destinadas exclusivamente a la docencia. Así lo afirman Daher y Sant'Anna (2010):

Nossa experiência nos permite observar, ainda, que as disciplinas dos cursos de licenciatura em Letras não costumam incorporar discussões sobre a formação para o trabalho do profissional professor de línguas estrangeiras, pois a maior parte das disciplinas desse curso preocupa-se com conteúdos sobre língua e literatura, ignorando ou não valorizando a relevância da articulação entre tal conteúdo e a atividade a ser realizada em situação de trabalho. (Daher y Sant'Anna, 2010, p. 64)

En este sentido, la percepción sobre lo que es ser profesor de lenguas está relacionado con la manera de cómo se entiende y se produce conocimiento en la Academia. A modo de ejemplificación, podemos pensar en cómo los estudios lingüísticos están vinculados con la forma tradicional de como se produce la ciencia. A principios del siglo XX, al transformar la lengua en objeto científico, los estudios de Saussure (2006 [1916]) acabaron por distanciar el sujeto de la lengua, con la finalidad de observarla y describirla. Más recientemente, sin embargo, viene ocurriendo un cambio paradigmático en el campo epistemológico, con el entendimiento sobre la continuidad entre sujeto y objeto (Santos, 1998, p. 13). Si la lengua nos constituye como sujetos, no hay cómo mirarla de fuera, sino de dentro.

De acuerdo con Daher y Sant'Anna (2010), una de las mayores dificultades encontradas en el campo de la enseñanza/aprendizaje de las lenguas, es el desequilibrio que hay entre la abundancia de trabajos que 
discuten acerca de la educación basada en métodos de aprendizaje de los alumnos y los pocos estudios dedicados a los conocimientos, que deben ser construidos junto a los profesores en formación inicial, para que estos puedan actuar profesionalmente a partir de las teorías trabajadas en los Profesorados. Considerando esto, defendemos que la formación de profesores de lenguas debe apoyarse más en la reflexión sobre diferentes miradas teóricas en concomitancia con la reflexión sobre la práctica docente, buscando el posicionamiento crítico, plural, contextualizado y socialmente comprometido con el cambio de paradigmas que sostienen las desigualdades.

Por lo tanto, con el fin de entender cómo se presenta el componente cultural en la formación del docente de portugués y español, tomamos como base los documentos oficiales de dos carreras de formación de profesores de idiomas de dos instituciones de educación superior, una en Brasil y otra en Argentina.

\section{Aspectos metodológicos y descripción de los contextos}

En el contexto brasileño, la carrera analizada fue la de Licenciatura em Letras ${ }^{4}$, de la Universidad Estadual Paulista. Este grado fue creado en 1959. La motivación para la selección de esta carrera es su prestigio en la formación docente y en los estudios lingüísticos de ámbito regional y nacional. En la actualidad, el grado de Letras tiene doble titulación, es decir, la formación para la enseñanza de: i) lengua portuguesa y sus literaturas; y, ii) un idioma extranjero y sus respectivas literaturas. Las opciones de idiomas que el grado dispone son Alemán, Español, Francés, Griego, Inglés, Italiano y Latín. La oferta de Lengua Española comenzó en 2005, convirtiéndose así en la última lengua extranjera en componer la matriz de la carrera. Es importante mencionar que en el mismo 2005 también fue sancionada en Brasil la Ley federal $n^{\circ} 11.161$, que dicta la obligatoriedad de la oferta de la lengua española en los currículos de la enseñanza secundaria brasileña.

De acuerdo con la página web de la institución, la carrera de Letras forma profesionales para el desarrollo de la investigación en las áreas de Lingüística, Lengua Portuguesa, Teoría de la Literatura Portuguesa y Brasileña, Filología Románica e Idiomas Extranjeros y sus Literaturas. Además, habilita para la actuación docente en la enseñanza básica. En este trabajo, nos cencomo profesor.

${ }^{4}$ En Brasil, el título de Licenciatura es el que habilita el profesional a actuar 
tramos en analizar las informaciones que corresponden a la habilitación en lenguas, especialmente la lengua española. Así, denominaremos la carrera como "Letras-Español".

Por otro lado, el grado de Profesorado de Portugués que también vamos a analizar, pertenece a la universidad más antigua de Argentina, la Universidad Nacional de Córdoba (UNC). Su creación data del año 1613, mientras que la oferta de lenguas en dicha institución comenzó en la década de 1920, cuando se creó el Departamento de Idiomas, perteneciente a la Facultad de Derecho y Ciencias Sociales. El foco del Departamento era la enseñanza de Francés, Italiano y nociones básicas de Latín jurídico. En el año 1926, el Departamento de Idiomas se convirtió en un Instituto de Idiomas, pasando a ofrecer las primeras carreras de formación de profesores, los Ilamados Profesorados. Las lenguas de habilitación eran el Alemán, el Francés, el Inglés y el Italiano.

Después de sucesivos proyectos para la formación de una Facultad de Lenguas en la UNC, no fue hasta el 2000 cuando se creó. En la actualidad, la Facultad de Lenguas de esta universidad cuenta con 17 carreras en las modalidades profesorado, licenciatura y traductorado público, con ofertas en las lenguas alemana, española (lengua materna y extranjera), francesa, italiana y portuguesa, lo que la convierte en una referencia para la formación del profesional de lenguas en Argentina.

El Portugués se ofrece sólo en el grado del profesorado, cuyo inicio fue en el año 2001. Por lo tanto, la lengua portuguesa fue la última en ser incorporada entre las opciones de la Facultad, al igual que el Español fue la última lengua que formó parte de las habilitaciones de la Unesp en Brasil.

La página web de la UNC, indica que el Profesor de Portugués (título dado a la persona que completa la carrera de Profesorado de Portugués), así como otros profesores de lenguas extranjeras formados por la Facultad "puede desempeñarse como profesor del idioma en los diferentes niveles de enseñanza, en establecimientos oficiales o privados como así también elaborar, dirigir, ejecutar y supervisar programas para la capacitación de adultos con fines específicos, en organismos oficiales y/o privados".

Para el análisis de los grados seleccionados, nos basamos en los documentos oficiales disponibles en la página web de las dos universidades presentadas. Para la carrera de Letras - Espanhol, usamos el Projeto Pedagógico (en adelante PP); y, para la carrera de Profesorado de Portugués, usamos el Plan de Estudios (PE). Tanto el PP como el PE son documentos que se aproximan como propósito, ya que presentan las ideas esenciales que fundamentan y orientan una carrera. Con el fin de establecer un parámetro 
de equivalencia de informaciones entre los dos contextos analizados, además de los dos documentos citados, disponemos también de los Programas de las asignaturas ofrecidas en la carrera de Letras-Español. Esto es porque el PP no describe las asignaturas de la carrera, mientras que el PE, sí presenta una síntesis de cada asignatura que compone la carrera de Profesorado de Portugués.

Por medio de identificación y análisis del uso del término "cultura" a lo largo de los documentos indicados, pretendemos comprender las representaciones de cultura en la formación del docente de lengua en esas carreras de grado. En este análisis, somos sensibles a las cuestiones como qué culturas, además de cuándo y cómo se las indican en esos documentos reguladores.

\section{Análisis del contexto brasileño: la carrera de Letras - Espanhol}

De acuerdo con el PP, la carrera de Letras tiene como objetivo formar sujetos para la comprensión de aspectos relativos al hombre y su naturaleza, en sus relaciones inter y extra personales, y cuyo foco sea "a linguagem humana, em seus múltiplos aspectos e desdobramentos, quais sejam, literários, comunicativos, imbricados com artes plásticas ou outras formas de expressão artística, e assim por diante" (UNESP, 2007, p. 1). En cuanto a la lengua extranjera moderna, se verifica que el objetivo de la carrera se centra en el desarrollo de las cuatro habilidades, conforme a lo presente en el documento: "no que se refere à formação em uma língua e literatura estrangeiras modernas, a graduação deve levar à aquisição de competência lingüística nas quatro habilidades, isto é, a produção escrita, a fala, a compreensão auditiva e a leitura" (UNESP, 2007, p. 5).

En cuanto al contenido curricular, la carrera está apoyada en dos ejes que son clásicos en la constitución de las carreras de Letras en Brasil: el campo de las Teorías Lingüísticas y el campo de las Teorías Literarias. Estos ejes están contemplados por las asignaturas ofrecidas en tres Departamentos, a saber: el Departamento de Letras Modernas, el de Lingüística y el de Teorías Literarias. Además, como se ha indicado, existe la parte de Licenciatura, cuya formación pedagógica está a cargo de los Departamentos de Ciencias de la Educación, de Didáctica y de Psicología de la Educación.

De este modo, ofrecemos, a continuación, el organigrama con una síntesis de las áreas de la carrera y su malla curricular: 

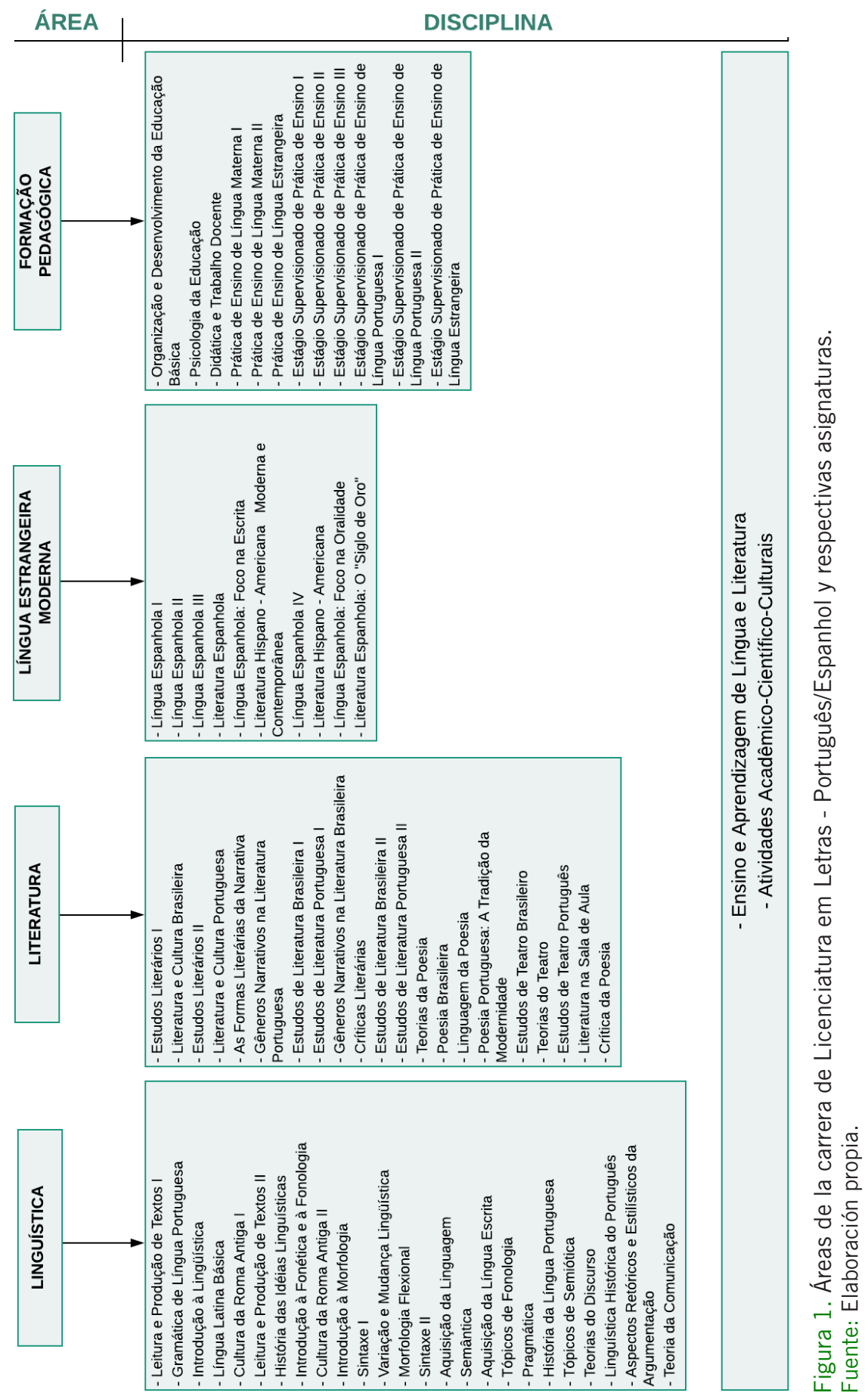
Específicamente en relación al componente cultural, en el PP, los términos "cultura(s)"/"cultural(is)" apareció 15 veces. En comparación con el PE de la carrera de Profesorado de Portugués, también analizado, "cultura(s)"/"cultural" fue identificado en 48 ocasiones. Así, podemos considerar que "cultura" y sus variantes son relativamente poco frecuentes en el documento de esa institución brasileña. Además, se debe considerar que en el documento brasileño las inserciones no vienen acompañadas de lo que se entiende por el término, a diferencia de lo que inicialmente sucede el currículo argentino.

En el documento brasileño, un aspecto que se debe analizar es el uso del término, que se hace ora en el singular, ora en el plural. Cuando se utiliza en el singular, "cultura" puede ser entendida de forma totalizante, homogénea, como, por ejemplo, en el nombre de la asignatura Cultura da Roma Antiga. Es importante subrayar que la única asignatura que presenta la palabra "cultura" en su título, es la que justo hace referencia a una civilización del pasado -Cultura de la Roma Antigua-, indicando de esta manera un conjunto de representaciones fijas sobre un determinado grupo social, geográficamente e históricamente situado, de carácter "valorativo-mítico" (Dussel, 2005). Por no tratarse de una asignatura específica del área de lengua y literatura española, no nos detendremos en analizar su Programa, pero vale adelantar que, así como no hay en el área de Español asignaturas en cuyo título esté explicitado "cultura", tampoco en los Programas de las asignaturas del área hay mención a ese término.

En la Introducción del PP, el término aparece dos veces, como se indica a continuación:

Uma vez que toda teoria necessita ser continuamente testada e aplicada com vistas à assimilação e, também, que necessita sempre de um corpus sobre o qual possa promover análises, a proposta filosófico-metodológica do Curso de Letras efetiva-se por meio de estudos de culturas específicas, antigas e contemporâneas, analisadas em suas manifestações lingüísticas e literárias, de modo que, enquanto a formação geral fornece as bases mais propriamente abstratas, necessárias para formar um sujeito crítico e capaz de empreender reflexão autônoma sobre a linguagem humana e suas manifestações literárias, (...) mas comprometidas com uma(s) cultura(s) particular(es), garantindo, ao mesmo tempo, as várias possibilidades de formação proporcionadas pelo Curso de Letras, de modo a proporcionar aos formandos, ao mesmo tempo, os conteúdos necessários para ocuparem as vagas do mercado de trabalho que a sociedade civil requisitar, tendo em vista suas necessidades de atuação profissional. (UNESP, 2007, p. 02, destacado nuestro) 
A partir de la lectura del fragmento anterior, es posible afirmar que, aunque exista el empleo de "culturas", en el plural, eso no implica la adopción de una perspectiva más alineada con una visión posmoderna del mundo, marcada por la descentralización del sujeto y en la formación de identidades fluidas -las identidades culturales (Hall, 2000)-. En otro sentido, el empleo del término en el plural se alinea más con la concepción relativista alemana de cultura, surgida en el siglo XVIII, cuyo foco se centraba en las particularidades de estados-nación. En el documento analizado, la asociación de adjetivos con el término, como "culturas específicas" y "culturas particulares", contribuye al efecto restrictivo, singularizando culturas, en vez de sugerir la ampliación y complejidad de diferentes identidades existentes dentro de una misma sociedad, tal como se espera en una perspectiva posmoderna.

En ese sentido, Salomão (2016), que también analizó este documento, pero con la finalidad de reflexionar sobre lengua y cultura en la formación de profesores de inglés, afirma que "o PP parece partir da visão de cultura modernista, de uma comunidade nacional um tanto quanto homogênea com suas instituições, costumes e modo de vida de longa data, enfocando um objetivo utilitarista de "ocupar vagas no mercado de trabalho"' (Salomão, 2016, p. 482).

Sólo cuando el documento trata de dos asignaturas que componen el campo de la Formación Pedagógica (Organização e Desenvolvimento da Educação Básica y Didática e Trabalho Docente), "cultura" es citada explícitamente:

A disciplina "Didática e Trabalho Docente" tem como objeto de estudo o professor e o ensino e centra-se na análise e na reflexão do trabalho e ação docentes no âmbito da sala de aula, da cultura escolar e do contexto sócio-econômico-político cultural da realidade brasileira, em que está inserida, buscando, desse modo, uma compreensão crítica e contextualizada do ensino e do trabalho docente. (...) Procura também fazer com que reflitam sobre a identidade, o papel, a intencionalidade e as práticas de ensino do professor, na formação dos alunos da escola fundamental e média. (UNESP, 2007, p. 10-11, destacado nuestro)

En esta situación, el término, repetido dos veces, da indicaciones de referirse al contexto educativo brasileño, en el sentido de situar la reflexión y práctica del futuro docente. El término "cultura", en este fragmento, es empleado para pensar el contexto educativo ("cultura escolar") y su entorno, referido como "contexto sócio-econômico-político cultural" y "condicionantes históricos, sócio-políticos e culturais". De tal forma, que la cultura aparece diluida entre otros términos, que determinan y especifican un grupo social, los sujetos que participan de la comunidad escolar, con foco en los estudiantes. 
Aunque la propuesta no explicite la reflexión sobre lenguas y culturas, sino la enseñanza a partir de la consideración de factores locales, consideramos que ese trecho ofrece elementos que se aproximan más a una reflexión decolonial de educación, al proponer formar al profesor para pensar críticamente el tema de la enseñanza desde una perspectiva local. La propuesta de diálogo entre paradigmas educativos y los "condicionantes históricos, socio-políticos y culturales" (UNESP, 2007, p. 10) abrirían posibilidades para una movilización de saberes que se desplazan y se resignifican.

En el documento, es recurrente la palabra "cultura" -ya sea en plural o en singular- seguida de otros términos. En cuatro momentos, el término viene yuxtapuesto a las palabras "académico" y "científico", con la denominación de actividades extras que componen la formación profesional, las Actividades Académico-Científico-Culturales (indicadas en el documento como seminarios, eventos científicos, artísticos y culturales, semanas de estudios, y otros):

Conta-se aí também, 15 outro conjunto de atividades acadêmico-cientifícoculturais a serem desenvolvidas durante a graduação, de modo a constituir a seguinte distribuição:

(...)

- Atividades Acadêmico-Científico-Culturais (freqüência a seminários, eventos científicos, artísticos e culturais, semanas de estudos, e outros. e elaboração de uma Monografia de Conclusão de Curso (MCC); " (UNESP, 2007, p. 14-15, destacado nuestro)

$\mathrm{y}$

na composição da carga horária total (...), serão computadas atividades de trabalho extraclasse, individuais ou em grupo, com ou sem tutoria, em iniciação científica, em estágios e prestação supervisionada de serviços à comunidade, etc. São as chamadas atividades acadêmico-científicoculturais. (UNESP, 2007, p. 18, destacado nuestro)

EI PP no presenta mayores indicaciones sobre lo que se encuadraría como evento cultural, pero da indicios de lo que sería por su diferenciación en relación a los dos términos que aparecen antes. Esto nos permite inferir, entonces, que lo "cultural" es lo que no es "académico" o "científico", aunque tenga relación con ellos por formar parte de un conjunto de actividades extras, previstas en un curso de licenciatura.

En relación a los Programas de Estudios, nos detendremos específicamente en las asignaturas que se refieren a la lengua española y sus literaturas, además de las prácticas de enseñanza en esa lengua. Verificamos que las asignaturas del área de Lengua Española se dividen en dos grupos, siendo uno para el campo de la lengua y otro para la literatura. Un aspecto a observar es que las asignaturas de literatura están divididas en dos ejes, la 
literatura española y la literatura hispanoamericana. La organización curricular enfatiza así esta idea ya cristalizada en la enseñanza de lengua española, en la que hay una contraposición entre España y la América española, con diferencias fundamentales y esencializadas, que permiten englobar una diversidad de literaturas bajo la definición de literatura hispanoamericana. Además, la organización por medio de la división igualitaria de créditos entre las "dos literaturas" contribuye a determinar el lugar minoritario que se da a las producciones literarias de los países hispanoamericanos.

Otro aspecto a ser evidenciado, es la delimitación cronológica de esas asignaturas del área de la literatura de lengua española. Nos Ilamó la atención el período seleccionado para cada "subárea" de la literatura. Cuando se refiere a la literatura de España, la carrera presenta una asignatura para discutir específicamente el Siglo de Oro, época comprendida entre los siglos XVI y XVII, conocida por la formación y ascenso del Imperio Español y marcada por el auge de la producción intelectual española -inaugurada por la publicación de la primera gramática de la lengua española en el siglo anterior de Antonio de Nebrija, en 1492)-. El triunfo cultural español, representado por medio de su literatura, es foco de estudio detallado en la carrera, que tiene como enfoque el estudio de autores como Lope de Vega, Calderón de la Barca y Miguel de Cervantes.

Ya en literatura hispanoamericana, se opta por desmenuzar la literatura moderna, que se inicia con el propósito de construir la idea de identidad nacional en los países hispanoamericanos por medio de los procesos de independencia desencadenados a partir del siglo XIX. Así, la asignatura busca comprender, de acuerdo con su Programa de Estudios, la "evolução da narrativa hispano-americana contemporânea", como se afirma en el propio documento, tomando como punto de partida el siglo XIX, es decir, desconsiderando trescientos años iniciales de historia literaria de la América española colonizada.

Vemos entonces una contraposición entre las dos subáreas. Por un lado, la asignatura de Literatura de España opta por revisar el pasado glorioso de la España colonial, que coincide con el apogeo y el declive del Imperio Español. De otro, la asignatura de Literatura Hispanoamericana dirige sus discusiones hacia las producciones literarias de esa región a partir del siglo XIX, período de formación de identidades nacionales. Lo que vemos, entonces, es el estudio de la literatura fuertemente vinculada a la idea de nación. Si consideramos que la perspectiva clásica alemana de "cultura" (que orienta la definición del término hasta hoy en el campo epistemológico occidental) surge con el propósito de formar una unidad nacional a partir de la promoción de características y producciones intelectuales locales, podemos Ilegar a la conclusión de que la Literatura de la carrera de Letras - Espanhol también 
organiza su literatura con base en ese principio, ya que selecciona contenidos que coinciden con períodos clave de la formación de las naciones hispanohablantes. Sobre el tratamiento de la cultura, podemos inferir, con base en esa reflexión sobre la presentación de la literatura, que los documentos tienden a definir cultura también a partir de una matriz clásica, con la construcción de la identidad de un pueblo por medio de su delimitación geográfica.

Las asignaturas del eje "Formación docente" se centran en la presentación de la historia de las políticas educativas, estructura de la educación básica, teorías del campo de la psicología de la educación y reflexión y práctica sobre el hacer docente. Por medio de su análisis, vemos que no hay ninguna mención a la cultura y a su práctica en el aula, ni como contenido, ni como propuesta de trabajo, aunque el término esté presente en la parte de la Formación Pedagógica del PP, como hemos comentado en este análisis.

\section{Análisis del contexto argentino: la carrera de Profesorado de Portugués}

De acuerdo con el PE del Profesorado de Portugués de la UNC, el profesor de portugués (título dado a la persona que completa este grado) será capacitado en las áreas de:

- lengua portuguesa, en sus niveles morfo-sintáctico, fonético y fonológico, pragmático y discursivo, con especial interés por las variantes del portugués moderno; lingüística aplicada a la enseñanza de la lengua y a la intercomprensión de lenguas romances; - de cultura, mediante el conocimiento de aspectos socio-culturales y literarios del mundo lusoparlante confrontados a idénticos aspectos de Argentina en general, con enfoque integrador, y formación docente general y especializada, mediante el conocimiento de las variables que intervienen en los procesos de enseñanza y aprendizaje. (UNC, s. f., p. 04)

Al final, el profesional deberá estar capacitado para "manejar técnicas de enseñanza, planificar y evaluar procesos de enseñanza-aprendizaje, y confrontar teorías y propuestas metodológicas y culturales" (UNC, s. f., p. 04). El enfoque a la cultura en la formación del profesional docente se hace presente, siendo ella uno de los tres pilares sobre el cual se estructura la carrera (a saber: áreas de Lengua, de Cultura y de Formación docente). El siguiente organigrama sintetiza los componentes que constituyen cada área de la carrera de Profesorado de Portugués: 

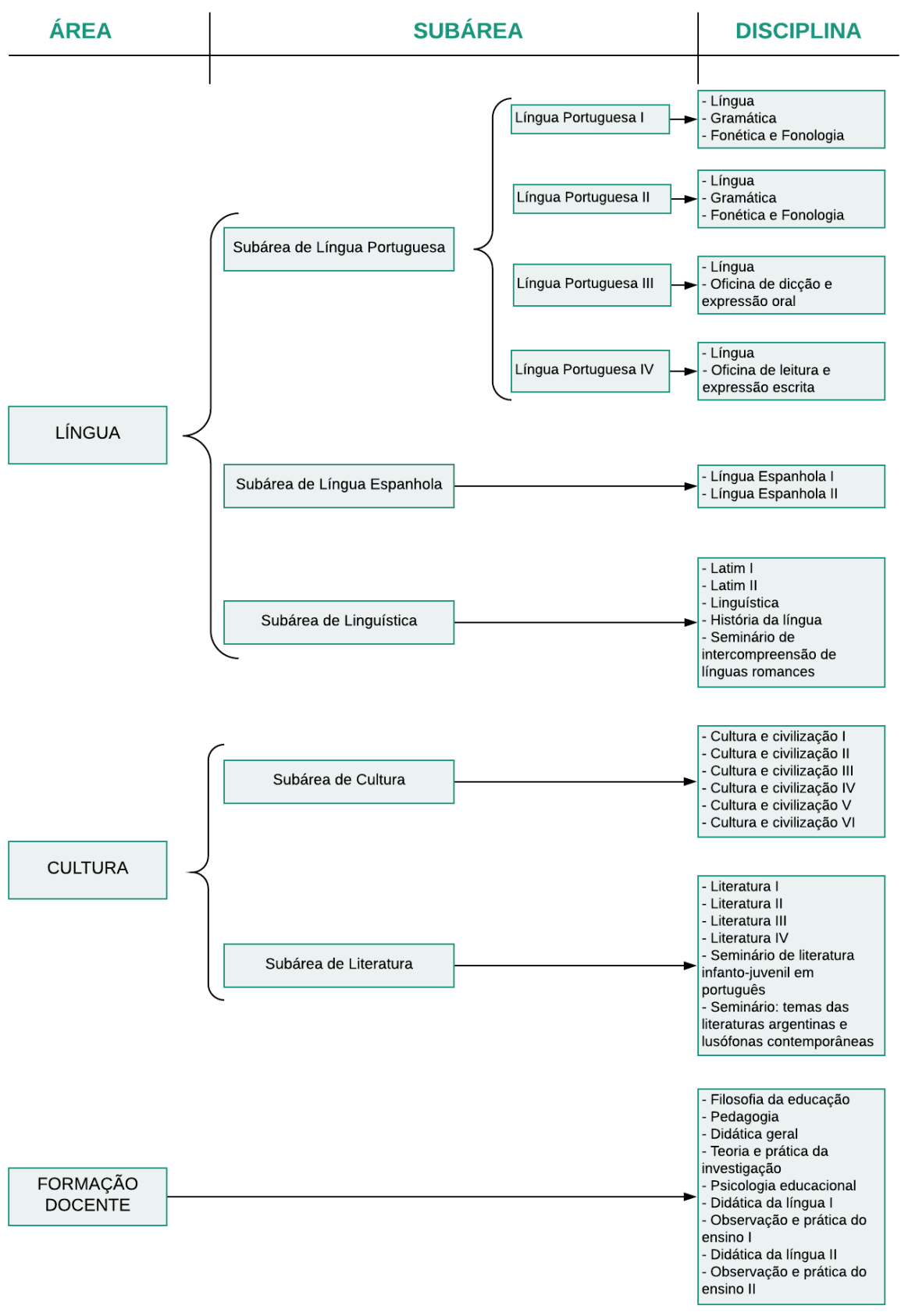

Figura 2. Áreas de la carrera de Profesorado de Portugués y respectivas subáreas y asignaturas.

Fuente: Elaboración propia. 
A partir de la lectura del ítem organización del PE, las áreas de cultura y formación docente hacen mención a la cultura como foco de estudio para la carrera docente, mientras que el área de Lengua no hace ninguna alusión directa al término. En concreto, el área de cultura toma como objeto de estudio los "diferentes discursos circulantes en las sociedades de habla portuguesa privilegiando la brasileña" (p. 07). La referencia al término "discurso" nos remite al entendimiento que Kramsch (2017) da para cultura. Para la teórica, la cultura se construye por medio de los significados que los grupos sociales dan a sus prácticas discursivas (Kramsch, 2017, p. 143, subrayado nuestro). Por discurso, la autora entiende ese concepto a partir de los presupuestos de Bajtín, para el cual el discurso debe ser entendido como la lengua en su uso práctico, asociada a su contenido ideológico (Zakir, 2015). En este sentido, podemos inferir que los "discursos circulantes", a los que el documento hace referencia, son las prácticas de lenguas socialmente situadas, que permitirán que el estudiante alcance "las bases para la comprensión de los elementos que se inscriben en la lengua como presupuestos o sobreentendidos y cuyo conocimiento se hace necesario tanto para el aprendizaje como para la enseñanza de la lengua extranjera" (UNC, s. f., p. 07).

Además, se afirma que el estudio discursivo del lenguaje contribuye a familiarizar a los estudiantes con "los orígenes étnicos, lingüísticos y culturales de los pueblos de habla portuguesa y de Brasil en particular" (UNC, s. f., p. 07). Se entiende, de esta forma, que el estudio de muestras de uso de la lengua sirven como base para el mapeo histórico de la formación de la lengua y de la sociedad lusohablante, con miras a la identificación de una cultura genuina, anclada en el pasado, capaz de justificar la dinámica social "proporcionándole [al estudiante] una visión global de su historia política, cultural y de la difusión de la lengua portuguesa y de la cultura lusófona en el mundo" (UNC, s. f., p. 07).

Por eso, es comprensible que no sólo la subárea cultura, sino también la de literatura integren esa área, ya que esa es entendida como medio para pensar lenguaje y sociedad, según lo afirmado en el pasaje:

Se incluye en esta área, la Literatura, espacio de expresión de la sociedad y vehículo de la lengua, costumbres y tradiciones, con el propósito de facilitar a los estudiantes los medios teórico-instrumentales para el análisis de textos en portugués y el abordaje de las diferentes manifestaciones de la literatura brasileña y lusófona en general. (UNC, s. f. 2007, p. 07)

Es importante observar el lugar dado a las culturas y variedades lingüísticas brasileñas a lo largo de todo el documento, justificado por la necesidad de integración con Brasil. Al respecto, el PE lo evidencia en su introducción: 
El profesorado de Portugués surge como respuesta a la demanda de una sociedad cada vez más integrada culturalmente con todos los países de Iberoamérica de los cuales uno, Brasil, se comunica en otra lengua. Es por ello que entendemos la urgencia en la preparación de este plan con el objeto de ampliar el panorama de los títulos que ofrece la Escuela Superior de Lenguas" (UNC, s. f., 2007, p. 01)

Según el documento, la integración de los países iberoamericanos ya está presente en el ámbito cultural, exigiendo la formación urgente de profesores de portugués con la finalidad de consolidar la política de integración regional ya en marcha. Esta estrategia es resultado de la celebración del Tratado de Asunción (1991), precursor del MERCOSUR, según afirma:

Este plan, diseñado con el fin primordial de responder a una necesidad estratégica con relación a la actual política cultural de integración internacional, nace para sustentar el título de profesor de portugués como lengua extranjera. Esta meta se encuentra directamente relacionada con la firma del Tratado de Asunción - 26 de marzo de 1991 - que diera inicio al Mercado Común del Sur (MERCOSUR) el cual contempla la búsqueda de formas de integración socioeconómica y la cooperación en el desarrollo científico y tecnológico incluyendo asimismo el intercambio y la complementación cultural como medios eficaces para elevar la calidad de vida de los habitantes de los países miembros. (UNC, s. f., p. 01)

El documento señala que la integración regional entre los países miembros, presupone que esa política de cooperación e intercambio entre países sea simétrica, de forma que las acciones educativas para la integración se realicen por todos los participantes del acuerdo. Esto significa entender que, en el caso de la enseñanza de idiomas, tanto la enseñanza del portugués debe ser el compromiso de los estados del MERCOSUR, que tienen el español como lengua oficial como, en contrapartida, el español lengua extranjera debe ser responsabilidad de la educación brasileña, según el trecho:

Interesados por la concreción de los citados objetivos y atendiendo a que la lengua es un vehículo de unión y conocimiento entre los pueblos del mundo, en nuestro ámbito iberoamericano, resulta imperiosa la formación de docentes de las lenguas habladas en los países signatarios del tratado: el portugués y el español. (UNC, s. f., p. 02)

En el PE de Profesorado de Portugués, la opción por el portugués de Brasil ya está indicada, en la Introducción, como la razón primordial para la existencia de la carrera. Por lo tanto, como es de esperar, el énfasis en las variedades lingüísticas y fundamentos de la cultura brasileña, por así decir, se hace presente en los contenidos programáticos de las asignaturas Cultura 
y Civilización, presentes en la subárea cultura. Es importante considerar que, excepto por el nombramiento de la asignatura, no hay otras referencias a la civilización a lo largo del documento, estando actualmente, incluso, ese término retirado del título de las asignaturas. A partir de ahí, podemos entender que cultura y civilización pueden ser tomadas como términos sinónimos, remitiendo de este modo a la definición de Tylor (1971 apud Cuche, 1996) sobre un conjunto de características adquiridas por el individuo en sociedad. Esta visión totalizadora y descriptiva para los términos es confirmada por la definición de cada asignatura de esta subárea, como vemos a continuación.

Las asignaturas Cultura y Civilización, ofrecidas semestralmente hasta el sexto período de la carrera, proponen el recorrido cronológico de hechos históricos de los países lusohablantes, con énfasis en Brasil. De este modo, a excepción del primer año, que presenta como temática general la relacionada con el turismo, la geografía, la política, la economía, la cultura, la sociedad y la vida privada en relación con los países de habla portuguesa, como vemos en secuencia:

\section{II.1.1. Cultura y Civilización I:}

Los países lusoparlantes: aspectos geográficos y turísticos.

\section{II.1.2. Cultura y Civilización II:}

Los países lusoparlantes: aspectos de la vida política, económica, social, cultural y privada. (UNC, s. f., p. 12)

En los demás semestres, se proponen temas considerados como clásicos de la historia de Brasil: feudalismo, navegaciones, mercantilismo, colonización, independencia, esclavitud, jesuitas, imperio, proclamación de la república y aristocracia agrícola. Así se puede leer en las descripciones de las asignaturas:

\section{I.1.3. Cultura y Civilización III:}

El paso del Feudalismo al Renacimiento y su relación con las grandes navegaciones desarrolladas por los íberos como clave de lectura para entender los antecedentes del descubrimiento del Brasil.

\section{II.1.4. Cultura y Civilización IV:}

De la Colonización a la Independencia. Intereses mercantilistas y colonialistas de Portugal. La esclavitud de indios y negros La presencia jesuítica en la colonia. Los movimientos que condujeron a la Independencia. Repercusiones en los movimientos artísticos y literarios.

\section{II.1.5. Cultura y Civilización V:}


El Brasil imperial en el contexto del panorama histórico mundial. La Independencia. El Romanticismo y sus reflejos en la vida cultural brasileña como el inicio de la valoración del nacionalismo brasileño Inicio de los movimientos internos que empiezan a forjar la proclamación de la República del Brasil.

II.1.6. Cultura y Civilización VI:

Brasil desde la proclamación de la República hasta nuestros días. Influencia de la aristocracia agrícola. Panorama de las transformaciones económicas, políticas, sociales y culturales. (UNC, s. f., p. 12)

Un último aspecto importante es la referencia a la asignatura Cultura y Civilización V. Según el Programa de Estudios de la asignatura, su foco está en la discusión sobre la formación de una identidad nacional en el período comprendido entre Brasil Imperio y Proclamación de la República. Esta idea se aproxima a la concepción clásica de cultura, formulada en el período de creación de los estados nación europeos, los cuales se formaron a partir de un territorio, una lengua y una cultura (Cuche, 1996).

En las asignaturas de Cultura y Civilización, la ausencia de referencia a cuestiones lingüísticas, sugiere que la cultura se trata de forma totalmente disociada de la lengua, enfocándose sólo en aspectos históricos, hechos y productos. La propuesta de la carrera, que presenta lengua y cultura como dos áreas separadas, contribuye a pensar la cultura como un apartado cuando se trata de trabajar en el campo lingüístico.

El área de formación docente también hace referencia a la cultura, con enfoque en la reflexión contextualizada de los aspectos socioculturales para ser considerados en la práctica docente del futuro profesor de lenguas. Se aproxima, de esta forma, a lo señalado en las asignaturas de formación docente del Projeto Pedagógico de la carrera de español, analizado en este trabajo, que trata el término bajo la óptica de la cultura escolar y de la cultura del aprendiz para pensar la práctica de enseñanza/aprendizaje contextualizada.

\section{Conclusión: formación del docente de lenguas y el (no) lugar de la cultura}

Este trabajo tuvo como objetivo comprender cómo la cultura es representada en las carreras de formación de profesores de portugués y español de lenguas extranjeras. Aunque brevemente, las discusiones realizadas a partir del análisis de los datos y orientadas por los textos teóricos del área 
nos dio indicaciones del alcance de la cultura en las diferentes áreas que componen las carreras, bien como qué cultura(s) (no) son seleccionadas como contenido para la formación profesional.

Por medio del análisis de los documentos de las dos carreras en cuestión, verificamos que estos difieren sustancialmente en relación al lugar dado a la cultura en la estructuración de las carreras. En el Projeto Pedagógico de Letras - Espanhol de la Universidad Estadual Paulista faltan indicaciones explícitas sobre qué entiende la carrera por cultura y cómo y en qué momentos deberá ella ser problematizada. La no presencia del término, sin embargo, trae indicios de cómo la carrera lo entiende en la formación docente. Además, la tradición de este grado, fuertemente marcado por los estudios lingüísticos, tiende a privilegiar la esfera lingüística, separándola de la esfera cultural. Que además, en los Programas de Estudio, al referirse al componente cultural, lo hace en singular, evidenciando una concepción totalizadora y homogeneizante del término, remitiendo a un conjunto de informaciones relacionadas con lugares genéricos que hacen uso de la lengua española ("universo hispánico" y "contextos hispanos").

Además de la escasez de ocurrencias para el término "cultura", identificamos la invisibilidad de América Latina, inexistente en el Projeto Pedagógico de la asignatura de Letras-Español. Eso, a su vez, refuerza el silenciamiento epistémico y cultural que se ha ejercido sobre los pueblos de esa región a lo largo de los últimos siglos. En ese sentido, propiciar la visibilidad cultural latinoamericana es más que buscar el equilibrio de saberes, es promover la justicia social tantas veces negligenciada.

El Plan de Estudios de Profesorado de Portugués de la Universidad Nacional de Córdoba, a su vez, tiene la recurrencia cultura como uno de los ejes de la carrera. Se indica que la cultura debe ser analizada por medio del estudio de los discursos circulantes en la sociedad y cita a Brasil como foco de estudio. Hemos encontrado que hay factores que contribuyen al enfoque en la lengua y cultura brasileñas, tales como cuestiones políticas, económicas y geográficas, que van hacia el discurso de aprendizaje del portugués brasileño como una forma de integración regional.

Las culturas presentes en el documento analizado se representan a partir de la concepción clásica. Aunque exista la presencia de los estudios de literatura y cultura de Portugal y de los países africanos lusohablantes, el tratamiento de la cultura en la carrera está orientada, en especial, a la trayectoria histórica de formación y consolidación de la nación brasileña. Además, las descripciones de las asignaturas de los ejes lengua y cultura muestran que esas dos esferas se representan como independientes en el contexto brasileño. 
A pesar de los abordajes más clásicos de la cultura en las carreras analizadas, podemos identificar también caminos posibles para el desarrollo de una reflexión cultural menos esencializada y más comprometida con la visibilidad de grupos socioculturales. Señalar el Projeto Pedagógico de la carrera brasileña que, en las disciplinas del área de formación pedagógica, se propone la discusión sobre el trabajo docente a partir del contexto socioeconômico-político y cultural, que es donde se ubica la práctica educativa, siendo fundamental para promover una reflexión crítica del papel efectivo de la docencia.

Por fin, consideramos que nuestra comprensión sobre los contextos seleccionados se construye a partir de diferentes contextos, que traen lecturas particulares sobre la referencia cultura a partir de sus perspectivas y retos teórico-prácticos para la formación de profesores. En este sentido, hay que considerar que el equipo docente de esas instituciones -que desempeñan las actividades pedagógicas y elaboran los currículos de la carrera- vienen de formaciones diversas, aunque dentro de las áreas de letras, lingüística, literatura y educación. Por eso, es importante enfatizar que los conceptos de cultura que componen las carreras son reflejo también de la confluencia de formaciones académicas y saberes de esos profesionales.

En ese sentido, forma parte de la investigación de la autora de este artículo dar secuencia a este trabajo y escuchar a otros actores, como los docentes de las carreras y los estudiantes, futuros profesionales. Esto permitirá conocer de forma más profundizada las complejidades que involucran el papel que la cultura viene desempeñando en la formación docente en contexto iberoamericano, así como las posibilidades para el abordaje crítico de la cultura en la formación docente.

\section{Referencias bibliográficas}

Argentina (2007). Lineamientos Curriculares Nacionales para la Formación Docente Inicial, Instituto Nacional de Formación Docente, Ministerio de Educación. Disponible en https://bit.ly/2zsA3Bz

Brasil (2002). Diretrizes para a Formação de Professores da Educação Básica, em cursos de nível superior, Diário Oficial da União de 18/1/2002, Seção 1, p. 31. Disponible en https://bit.ly/1DB74aZ

Candau, V. M. (2008a). Direitos humanos, educação e interculturalidade: as tensões entre igualdade e diferença. Revista Brasileira de Educação, 13(37).

Candau, V. M. (2008b). Multiculturalismo e educação: desafios para a prática pedagógica. Moreira, A. F.; Candau, V. M. Multiculturalismo: Diferenças culturais e práticas pedagógicas. Petrópolis, Rio de Janeiro: Vozes. 
Cuche, D. (1996) A noção de cultura nas Ciências Sociais. Bauru: EDUSC.

Daher, D. C., y Sant'Anna, V. L. A. (2010). Formação e exercício profissional de professor de língua espanhola: revendo conceitos e percursos. Barros, C. S. de; Goettenauer, E. Espanhol: ensino médio. Organizado por Barros e Goettenauer de Marins Costa. (Coleção Explorando o Ensino). Brasília: Ministério da Educação. Secretaria de Educação Básica, 16, 55-68.

Dumas, M. L. S. (2010). Convênios de Cooperação e Intercâmbios: novos atores, novos cenários. Congresso Internacional de Professores de Línguas Oficiais do MERCOSUL. Foz do Iguaçu.

Dussel, E. (2005). Transmodernidad e Interculturalidad: Interpretación desde la Filosofía de la Liberación. Lander, E. (org.) La colonialidad del saber: Eurocentrismo y ciencias sociales. Perspectivas latinoamericanas. Buenos Aires: Unesco, Ciccus, Clacso.

Hall, S. (2000). Quem precisa de identidade? Silva, T. T. (org e trad.) Identidade e diferença: a perspectiva dos estudos culturais. Petrópolis: Vozes, 103-133.

Kramsch, C. (2017). Cultura no ensino de língua estrangeira. Bakhtiniana, Rev. Estud. Discurso, São Paulo , 12(3), 134-152. Recuperado de https://bit.ly/330pPcB

Moreira, A. F. B. (2001). A recente produção científica sobre currículo e multiculturalismo no Brasil (1995-2000): avanços, desafios e tensões. Revista Brasileira de Educação, $18,65-81$.

Salomão, A. C. B (2016). Língua e cultura no curso de graduação em Letras: uma reflexão sobre as concepç̃̃es de alunos, Projeto Político Pedagógico e programas de ensino. Estudos Linguísticos, 45, 474-490.

Santos, B. S. (1997). Uma concepção multicultural de direitos humanos. Contexto Internacional, 23, 7-34. Recuperado de https://bit.ly/2jwl5kL

Saussure, F. de. (2006 [1916]). Curso de linguística geral. 27. Cultrix: Ed. São Paulo.

UNC (s.f.). Plan de Estudios del Profesorado de Portugués. Facultad de Lenguas, Universidad Nacional de Córdoba, Argentina. Disponible en https://bit.ly/2ZuBMou.

UNESP (2007). Projeto Pedagógico do curso de Letras. Faculdade de Ciências e Letras do campus de Araraquara, Universidad Estadual Paulista, Brasil. Disponible en https:// bit.ly/31XMEIW

Zakir, M. de A. (2015) Cultura e $(m)$ telecolaboração: uma análise de parcerias de teletandem institucional. Tese (Doutorado em Linguística) - Universidade Estadual Paulista Júlio de Mesquita Filho. São José do Rio Preto. 